\title{
Philonsorbonne
}

14 | 2020

Année 2019-2020

\section{Un mythe luthérien de l'intériorité ? La grammaire wittgensteinienne du cœur}

\section{Mathieu EYCHENIÉ}

\section{(2) OpenEdition}

Journals

Édition électronique

URL : https://journals.openedition.org/philonsorbonne/1547

DOI : 10.4000/philonsorbonne. 1547

ISSN : 2270-7336

Éditeur

Publications de la Sorbonne

Édition imprimée

Date de publication : 14 février 2020

Pagination : 203-220

ISSN : 1255-183X

\section{Référence électronique}

Mathieu EYCHENIÉ, "Un mythe luthérien de l'intériorité ? La grammaire wittgensteinienne du cœur », Philonsorbonne [En ligne], 14 | 2020, mis en ligne le 15 mars 2020, consulté le 09 juin 2021. URL: http://journals.openedition.org/philonsorbonne/1547; DOI : https://doi.org/10.4000/philonsorbonne. 1547

(c) Tous droits réservés 


\title{
Un mythe luthérien de l'intériorité ? La grammaire wittgensteinienne du cœur
}

\author{
Mathieu EYCHENIÉ
}

Dans les conférences prononcées à Dartmouth College en novembre 1980, rassemblées dans l'ouvrage L'Origine de l'herméneutique de soi, Foucault distingue deux figures du souci de soi : le souci gréco-romain et le souci chrétien, qui se distingue du premier en ce qu'il est fondamentalement motivé par la découverte et l'expression de la vérité au sujet de soi, via notamment la place prépondérante accordée à la technique de l'aveu. Foucault voit dans le souci de soi chrétien la naissance de l'herméneutique de soi, au sens où le soi chrétien est « quelque chose que l'on doit découvrir ou déchiffrer comme un texte très obscur $»^{1}$. Ainsi «[c]hacun, chaque chrétien, a le devoir de savoir qui il est, ce qui se passe en lui ; il doit connaître les fautes qu'il peut avoir commises; il doit connaître les tentations auxquelles il est exposé. Et, en outre, chacun dans le christianisme est obligé de dire ces choses à d'autres personnes et donc de porter témoignage contre lui-même $»^{2}$.

Le domaine ainsi dévoilé, ainsi découvert, porte un nom : il correspond au motif biblique du cœur. Le croyant est appelé à « faire toute la vérité » sur lui-même, c'est-à-dire à manifester - devant les autres hommes, mais aussi et surtout devant Dieu - non pas seulement ce qu'il est, mais celui qu'il est, par le biais d'un témoignage porté contre lui-même (la mise au jour des fautes qu'il a commises et projette de commettre, des tentations auxquelles il se trouve exposé). Le décèlement du cœur prend ainsi la forme d'un approfondissement de soi : le croyant est enjoint de dévoiler ses

1. Michel Foucault, L'Origine de l'herméneutique de soi. Conférences prononcées à Dartmouth College, 1980, Paris, Vrin, 2013, p. 50.

2. Ibid., p. 67. 
pensées les plus intérieures, les plus intimes ${ }^{3}$, et de les confronter à ce qui, en lui, l'accuse ou le disculpe : sa conscience, au sens moral ${ }^{4}$. En me mettant à nu, je m'apparais à moi-même comme un être responsable - comme appelé à répondre, à chaque instant, de mes pensées et de mes actes.

Le croyant se doit ainsi de respecter une première obligation de vérité : dire la vérité sur soi-même. Une telle obligation, cependant, n'est pas autosuffisante; selon Foucault, elle se trouve intimement mêlée, dans le christianisme, à une seconde: adhérer à la vérité du Livre - une vérité révélée qui, historiquement, a pris la forme du dogme. La forme chrétienne de l'ascèse de vérité «a [ainsi] suscité deux types différents de relation: relation à la vérité révélée, à travers le Logos, à travers la Parole, à travers le Livre. Relation à la vérité en tant qu'elle est révélée à travers les figures du texte. [...] Et il y a une autre relation à notre réalité intérieure, à une réalité qui est cachée et enfouie dans les profondeurs de notre cœur, dans le secret de notre conscience, dans les mouvements à peine perceptibles de notre pensée $»^{5}$.

À suivre Foucault, le souci de soi chrétien possède donc deux caractères principaux : il oblige d'une part à un déchiffrement de soi, d'autre part à une adhésion sans réserve à un complexe de vérités - étant entendu que ces deux injonctions ne sont pas autonomes, mais renvoient l'une à l'autre.

Si le détail des analyses de Foucault peut sans doute être critiqué, ou, à tout le moins, amendé, ces dernières ont le mérite de dessiner les contours d'une intériorité ou d'un intérieur spécifique : l'intériorité du cœur. Pour insigne qu'elle soit, elle apparaîtra certainement à un œil wittgensteinien comme digne de soupçon : on dira en effet, spontanément, qu'elle opère une mythification, au même titre par exemple que le mentalisme cartésien auquel le « mythe de l'intériorité » est communément rapporté.

On ne manquera pas de noter, cependant, que le cœur chrétien se distingue radicalement de l'âme cartésienne. La naissance chrétienne de l'herméneutique de soi a en effet pour corollaire paradoxal la destruction du soi. Celui-ci se voit, dans un même mouvement, plus amplement découvert et profondément anéanti : au moment même où il dit la vérité sur lui-même, le croyant est appelé à renoncer au texte ainsi déchiffré. L'exomologesis (une technique particulière de pénitence, mentionnée par les Pères grecs)

3. Pour cette intériorité spécifiquement chrétienne, et le sens général de la notion de cœur, cf. le Vocabulaire de la théologie biblique, publié sous la direction de Xavier Léon-Dufour et Jean Duplacy, Augustin George, Pierre Grelot, Jacques Guillet et Marc-François Lacan, Paris, Éditions du Cerf, 1962, p. 136 : «Les résonances qu'éveille le mot “cœur” ne sont pas identiques en hébreu et en français. Dans notre façon de parler, le cœur est lié à la vie affective : c'est le cœur qui aime ou déteste, qui désire ou qui craint ; mais pour l'activité intellectuelle aucun rôle ne lui est attribué. L'hébreu parle du cœur en un sens beaucoup plus large. Le cœur, c'est ce qui se trouve tout au-dedans; or, à l'intime de l'homme, il y a certes les sentiments, mais aussi les souvenirs et les pensées, les raisonnements et les projets ».

4. En allemand : Gewissen, à distinguer de Bewußtsein.

5. Michel Foucault, Dire vrai sur soi-même. Conférences prononcées à l'Université Victoria de Toronto, 1982, Paris, Vrin, 2017, p. 141. 
manifeste tout spécialement ce double mouvement paradoxal, en donnant lieu à un énoncé radicalement anticartésien : « La formule qui est au cœur de l'exomologesis est [...] ego non sum ego. L'exomologesis cherche [...] à superposer, par un acte de rupture violente, la vérité au sujet de soi et le renoncement à soi. Dans les gestes ostentatoires de macération, la révélation de soi dans l'exomologesis est, au même moment, destruction de soi » ${ }^{6}$.

Là où le croyant, aux premiers temps du christianisme, met radicalement ses pensées en question jusqu'à soupçonner qu'il n'en est pas l'auteur (ne serait-ce pas le démon qui pense en moi ? ${ }^{7}$ ), la formulation même du cogito suppose, au début de la Méditation seconde, le rejet de cette possibilité :

[Q]ue sais-je s'il n'y a point quelque autre chose différente de celles que je viens de juger incertaines, de laquelle on ne puisse avoir le moindre doute ? N'y a-t-il point quelque Dieu, ou quelque autre puissance, qui me met en l'esprit ces pensées? Cela n'est pas nécessaire; car peut-être que je suis capable de les produire de moi-même [forsan ipsemet illarum author esse possim]. Moi donc à tout le moins ne suis-je pas quelque chose [Nunquid ergo saltem ego aliquid sum] $?^{8}$

Le cœur n'est pas l'ego. Dans la perspective du second Wittgenstein', le premier concept semble néanmoins charrier, au même titre que le deuxième, une illusion: faire de la croyance religieuse une "affaire de cœur » reviendrait à réifier, hypostasier un certain contenu ou intériorité psychologique - nos pensées les plus intimes, rapportées à la conscience. Un tel rejet paraît impliqué par cette remarque extraite des Leçons sur la croyance religieuse: "Une croyance n'est pas analogue à un état d'esprit momentané. "À cinq heures, il a eu un très fort mal de dents" »" ; ainsi que par le paragraphe 587 des Recherches philosophiques :

Y a-t-il un sens à demander: «D'où sais-tu que tu crois cela ?»-, et la réponse est-elle: "L'introspection me l'apprend [Ich erkenne es durch Introspektion] » ? Dans un certain nombre de cas, mais non dans la plupart, on pourra dire quelque chose de ce genre. Il y a un sens à demander : «Est-ce que je l'aime vraiment, ou est-ce que je me l'imagine seulement? », et le processus de l'introspection consiste à évoquer des souvenirs, à se représenter des situations possibles, et à imaginer les sentiments que l'on aurait si... ${ }^{11}$

6. M. Foucault, L'Origine de l'herméneutique de soi, op. cit., p. 74.

7. Cf. pour ce point ibid., p. 79-81.

8. Descartes, Méditations métaphysiques, Paris, Flammarion, 1992, p. 72-73 ; AT, VII, 24 (pour le latin) ; $A T$, IX, 19 (pour le français).

9. Auquel nous nous limiterons dans cet article.

10. Ludwig Wittgenstein, "Leçons sur la croyance religieuse », in Leçons et conversations sur l'esthétique, la psychologie et la croyance religieuse, suivies de Conférence sur l'Éthique, traduction par J. Fauve, Paris, Gallimard, 1992, p. 109.

11. Ludwig Wittgenstein, Recherches philosophiques, traduction par F. Dastur, M. Élie, J.-L. Gautero, D. Janicaud et É. Rigal, Paris, Gallimard, 2004, p. 219; Philosophische Untersuchungen, Frankfurt am Main, Suhrkamp Verlag, 2003, p. 249. 
Dans la plupart des cas, je ne sais pas que je crois à la suite d'une introspection: je réponds immédiatement à la question. Mais même si je procède à une introspection, celle-ci ne consiste pas en la contemplation d'un contenu psychologique, mais en différentes pratiques : l'évocation de souvenirs, l'imagination de situations possibles, etc.

D'un autre côté pourtant, une étude plus approfondie des textes wittgensteiniens traitant de la croyance religieuse montre que l'auteur des Recherches philosophiques fait reposer celle-ci, prioritairement, sur la foi. Il semble donc, dans les termes de Foucault, accorder un privilège à la première obligation de vérité (le déchiffrement du cœur) par rapport à la seconde ; ou pour mieux dire : il fait reposer la croyance sur la foi, partant sur ce qui - dans le cœur - fait le lien entre les deux obligations de vérité, entre le déchiffrement de soi et l'adhésion à la vérité révélée. Selon Wittgenstein, la révélation seule ne peut me conduire à croire: je crois au Livre parce que je suis intérieurement - par ma conscience - conduit à croire. Il l'indique clairement dans ses Carnets de Skjolden:

Dans la Bible, je n'ai rien qu'un livre devant moi. Mais pourquoi dis-je « rien qu'un livre»? J'ai un livre devant moi, un document qui, s'il reste seul, ne peut avoir plus de valeur que n'importe quel autre document. (C'est ce que Lessing $^{12}$ a pensé.) Ce document en soi ne peut m' « obliger » à croire aux leçons qu'il renferme, aussi peu que n'importe quel autre document, qui aurait pu me tomber entre les mains. Si je dois croire à ces leçons, ce n'est pas parce que cela et non pas autre chose m'a été rapporté. Au contraire, elles doivent me sauter aux yeux [...]. Ce n'est pas l'Ecriture, mais ma conscience [das Gewissen] qui peut me commander - de croire à la résurrection, au Jugement dernier, etc. Croire, non pas comme à quelque chose de vraisemblable, mais en un autre sens. Et mon incroyance ne peut m'être reprochée que si, ou bien ma conscience commande la foi [mein Gewissen den Glauben befiehlt] - s'il y a quelque chose de tel - , ou si elle me reproche des bassesses; ce qui, d'une certaine façon, mais que je n'ignore pas, ne m'amène pas à la foi. Cela signifie, me semble-t-il, que je dois dire : Tu ne peux absolument rien savoir maintenant d'une telle foi, elle doit être un état d'esprit [ein Geisteszustand] dont tu ne sais absolument rien et qui ne te concerne en rien aussi longtemps que ta conscience [Gewissen] ne t'en apporte pas la révélation; au contraire il te faut maintenant suivre ce que te dit ta conscience [Gewissen]. [...] Ce qui est prêché peut servir de préliminaire à la foi, mais cela ne peut vouloir l'animer grâce à ce qui s'y annonce. ( $\mathrm{Si}$ ces paroles pouvaient obliger à croire, d'autres paroles le pourraient aussi). La foi commence avec le fait de croire [Das Glauben fängt mit dem Glauben an]. On doit commencer avec la foi; des paroles ne résulte aucune foi ${ }^{13}$.

12. Wittgenstein fait probablement référence ici à l'ouvrage Die Erziehung des Menschengeschlechts.

13. L. Wittgenstein, Carnets de Cambridge et de Skjolden, 1930-1932, 1936-1937, traduction par J.-P. Cometti, Paris, Presses universitaires de France, p. 98-99 ; MS 183, 148-151 [1937]. Cf. également pour le même point L. Wittgenstein, Remarques mêlées, traduction par G. Granel, Paris, Flammarion, 2002, p. 132-133 ; Vermischte Bemerkungen, Frankfurt am 
La croyance religieuse apparaît bien ici comme une « affaire de cœur» : elle dépend tout entière d'une pensée intime - la foi - à laquelle je dois, intérieurement, me résoudre, me décider.

Le second Wittgenstein paraît donc, tout à la fois, mettre en garde contre l'hypostase du cœur et faire de la religion une question intime, intérieure. Nous tenterons, dans cet article, de dépasser cette contradiction apparente, en mettant au jour ce que nous appellerons la grammaire wittgensteinienne du cœur. Il s'agira, pour le dire autrement, de décrire les motifs et figures qui composent la «tapisserie » de la vie religieuse - en tant que pratique intérieure, ou pratique de l'intériorité. Nous étudierons, pour ce faire, les rapports que la philosophie wittgensteinienne de la religion entretient avec la tradition théologique qui a mis la foi au centre de la vie religieuse : la tradition luthérienne.

\section{1. «[L]a foi se trouve sous la mamelle gauche»}

Wittgenstein mentionne relativement peu Luther. On note toutefois, immédiatement, un certain " air de famille», une certaine proximité de vue entre les deux auteurs qui se manifeste dans leur méfiance commune vis-àvis du pouvoir ecclésiastique.

L'auteur des Recherches philosophiques identifie souvent l'autorité ecclésiastique à la figure de Paul. Il note ainsi dans une de ses Remarques mêlées :

La source qui, dans les Évangiles, coule transparente et claire, semble écumer dans les Epîtres de Paul. Du moins cela me semble à moi. Peut-être estce seulement ma propre impureté qui voit en elles quelque chose de trouble ; car pourquoi cette impureté ne pourrait-elle point souiller la clarté ? Mais pour moi, c'est comme si je voyais ici la passion humaine, quelque chose comme l'orgueil ou la colère, qui rime mal avec l'humilité des Évangiles. Comme s'il y avait bel et bien ici une insistance sur sa propre personne, et ce en tant qu'acte religieux, chose tout à fait étrangère à l'Évangile. [...] Dans les Évangiles -

Main, Suhrkamp Verlag, 1977, p. 122-123 [1947]: «Il me semble qu'une foi religieuse pourrait n'être qu'une sorte de décision passionnée en faveur d'un système de référence [etwas wie das leidenschaftliche Sich-Entscheiden für ein Bezugssystem]. Que, par conséquent, bien que ce soit une foi, c'est cependant une manière de vivre [eine Art des Lebens], ou une manière de juger la vie [eine Art das Leben zu beurteilen]. Instruire quelqu'un dans une foi religieuse devrait donc consister en l'exposition, la description du système de référence, et en même temps en un appel à la conscience [ein ins-Gewissenreden]. Ces deux aspects de l'instruction devraient avoir finalement pour effet que celui qui la reçoit embrasse lui-même, de son propre mouvement, avec passion le système de référence en question. À peu près comme si quelqu'un me faisait voir d'un côté combien ma situation est désespérée, tandis que de l'autre côté il me montrerait l'instrument du salut, jusqu'à ce que de moi-même, ou en tout cas sans que mon instructeur ne me conduise par la main, je me jette sur cet instrument et m'en saisisse ». 
c'est ce qu'il me semble - tout est plus simple, plus humble. On se trouve là comme dans une chaumière; chez Paul, on trouve une Église. Là tous les hommes sont égaux, et Dieu même est un homme ; chez Paul, il y a déjà quelque chose comme une hiérarchie, des dignités et des charges ${ }^{14}$.

La vie claire et limpide des premiers chrétiens s'est trouvée obscurcie par les épîtres pauliniennes, qui annoncent l'instauration des premières églises, partant des premiers rapports d'autorité. Certes Wittgenstein a évolué dans sa lecture de Paul : il affirme, au cours de ses conversations avec Maurice Drury, que s'il a effectivement tenu à une époque les Évangiles et les Épitres pour des textes issus de religions différentes, il s'est ravisé au cours des années suivantes ${ }^{15}$. Reste qu'il n'a pas renoncé à sa méfiance visà-vis des autorités ecclésiastiques. La croyance religieuse ne saurait être imposée par aucune instance, aucun pouvoir extérieur : elle ne peut procéder, nous dit très précisément Wittgenstein, que du cœur. Ainsi par exemple concernant le jeûne : " Je veux le faire, si cela me vient du cœur [wenn es mir von Herzen kommt] \& non pas parce que cela m'est ordonné $»{ }^{16}$.

Plus exactement, nous l'avons dit, Wittgenstein rapporte prioritairement la croyance religieuse à la foi. Celle-ci doit être nettement distinguée de l'intelligence ou de la sagesse. Elle procure une certitude - la certitude du salut - qui n'est pas d'ordre intellectuel ou spéculatif: " $\mathrm{Si}$ je dois réellement être sauvé, alors c'est la certitude [Gewißheit] qu'il me faut, non la sagesse [Weisheit], les rêves, la spéculation - et cette certitude est la Foi [der Glaube]. La Foi est foi en ce que demande mon cour [Herz], mon âme [Seele], non mon entendement spéculatif [spekulierender Verstand] ${ }^{17}$.

La croyance chrétienne ne consiste pas en une opinion : en ce sens, le croyant fait un usage insigne du mot $\langle\text { croire }\rangle^{18}$. Cela revient à distinguer la croyance religieuse du domaine, plus général, de la science - c'est-à-dire précisément de l'intelligence ou de la sagesse. La croyance religieuse n'est affaire, plus exactement, ni de vérités rationnelles ni de vérités historiques, c'est-à-dire de vérités de fait :

14. L. Wittgenstein, Remarques mêlées, op. cit., p. 89 ; Vermischte Bemerkungen, op.cit., p. 64 [1937].

15. Cf. Maurice Drury, Conversations avec Ludwig Wittgenstein, traduction par J.-P. Cometti, Paris, Presses universitaires de France, 2002, p. 190 : «Il fut un temps où je pensais que les Épîtres de Paul étaient d'une religion différente de celle des Évangiles. Mais maintenant, je vois clairement que j'avais tort. Dans les Évangiles et dans les Épîtres, on a bien affaire à une seule et même religion "».

16. L. Wittgenstein, Carnets de Cambridge et de Skjolden, op. cit., p. 136 ; MS 183, 225 [1937].

17. L. Wittgenstein, Remarques mêlées, op. cit., p. 93 ; Vermischte Bemerkungen, op. cit., p. 69 [1937].

18. Pour une analyse détaillée de ce point, cf. Élise Marrou, «De la certitude religieuse : Wittgenstein sur la corde raide », ThéoRèmes [En ligne], 1/2011, mis en ligne le 15 décembre 2011. 
Le christianisme ne se fonde pas sur une vérité historique, il nous donne un récit (historique), et dit : maintenant, crois! Non pas : accorde à ce récit la foi [glaube diese Nachricht mit dem Glauben] qui convient à un récit historique, mais : crois quoi qu'il arrive, ce qui ne peut être que le résultat d'une vie [Resultat eines Lebens]. Tu as là un récit - Ne te comporte pas envers lui comme envers les autres récits historiques! Donne-lui une place tout autre dans ta vie. [...] La preuve historique (le jeu de la preuve historique) ne concerne pas la foi. Ce récit (les Évangiles) a été reçu par les hommes avec foi (c'est-à-dire avec amour). C'est cela, et rien d'autre, qui fait la sûreté de ce tenir-pour-vrai. Le croyant n'a envers ce récit $n i$ la relation que l'on a envers la vérité historique (la vraisemblance), $n i$ celle que l'on a envers une doctrine des vérités rationnelles ${ }^{19}$.

Le croyant n'est pas pour autant un fou. Certains passages de Wittgenstein semblent certes plaider pour l'assimilation de la foi à une forme de folie; ainsi notamment cette remarque des Leçons sur la croyance religieuse, dirigée contre le Père O'Hara qui tend à confondre religion et science : «C'est ce que disent les Épîtres qu'il suffit de lire : non seulement ce n'est pas raisonnable, mais c'est folie $»^{20}$. Wittgenstein fait ici clairement référence à l'inversion des valeurs manifestée par la crucifixion, la mort de Dieu : la sagesse de ce monde est devenue folie aux yeux de Dieu, selon la formule de la première Épitre aux Corinthiens, 1, 2012. Dans d'autres passages, Wittgenstein relève également certains traits communs à la folie et la croyance : en particulier la souffrance du croyant, couplée à un sentiment d'isolement, que la religion a cependant pour tâche d'éliminer ${ }^{22}$.

Aux yeux de Wittgenstein, la croyance chrétienne n'est toutefois pas une folie au sens strict, dans la stricte mesure où elle n'est pas, à proprement parler, irrationnelle - cela parce qu'elle relève d'un autre ordre que la raison ou la science. Le concept de Dieu, par exemple, est potentiellement

19. L. Wittgenstein, Remarques mêlées, op. cit., p. 91-92 ; Vermischte Bemerkungen, op. cit., p. 67-68 [1937].

20. L. Wittgenstein, «Leçons sur la croyance religieuse », op. cit., p. 114.

21. Cf. La Bible de Jérusalem, traduction sous la direction de l'École biblique de Jérusalem, Paris, Éditions du Cerf, 1998, p. 1920 : «Dieu n'a-t-il pas frappé de folie la sagesse du monde? ».

22. Cf. sur ce point, notamment, L. Wittgenstein, Remarques mêlées, op. cit., p. 108-109 [environ 1944] : «Il n'y a pas de plus grand cri de détresse que celui d'un homme seul. [...] La religion chrétienne n'est faite que pour celui qui a besoin d'un secours infini, c'est-à-dire pour celui qui éprouve une détresse infinie. [...] Celui à qui il est donné, au sein d'une telle détresse, d'ouvrir son cœur au lieu de se rétracter sur lui-même, celui-là accueille le remède dans son cœur. Celui qui ouvre ainsi son cœur à Dieu dans la contribution de la confession, celui-là s'ouvre aussi aux autres. [...] On pourrait dire aussi bien : la haine entre les hommes provient de ce que nous nous coupons les uns des autres, et cela parce que nous ne voulons pas que l'autre regarde en nous, où il n'y a rien de beau à voir. Cela dit, si nous devons continuer à avoir honte de ce qui est en nous, nous ne devons pourtant pas avoir honte devant nos compagnons d'humanité ». 
compréhensible : non pas cependant parce qu'il aurait un contenu rationnel que je pourrais saisir, mais parce qu'il engage une manière de vivre - la foi que je peux adopter, que je peux faire mienne. Ainsi «[1]a religion en tant que folie [Religion als Wahnsinn] est une folie qui provient de l'irréligiosité ${ }^{23}$. La foi requise par la religion chrétienne n'est absolument pas de l'ordre de la raison; croire ne consiste donc pas à tenter d'intellectualiser une série de paradoxes ${ }^{24}$, qui se présentent comme autant d'irritations ou chatouillis de l'intellect :

Je crois : le mot «croire », dans la religion, a fait effroyablement de mal. Toutes les pensées alambiquées sur le «paradoxe», la signification éternelle d'un fait historique et autres choses semblables. Mais si, au lieu de dire « La foi en Jésus-Christ », tu dis : " Amour du Christ », le paradoxe disparaît, c'est-àdire l'irritation de l'intellect [die Reizung des Verstandes]. Qu'est-ce que la religion a à voir avec une chatouille de l'intellect [mit so einem Kitzeln des Verstandes]. (Cela peut aussi faire partie de la religion de tel ou tel.) $\mathrm{Ce}$ n'est pas que l'on puisse dire maintenant : Oui, maintenant, tout est simple ou compréhensible. Ce n'est en rien compréhensible, ce n'est seulement pas incompréhensible $^{25}$.

L'auteur des Recherches philosophiques semble distinguer deux niveaux de compréhension des concepts religieux. À un premier niveau, je les comprends au sens où je reconnais les mots qui sont employés et où je peux les utiliser dans certains contextes (par exemple dans un récit). Wittgenstein fait toutefois également un autre usage du terme «comprendre »: il distingue cette première espèce de compréhension

23. L. Wittgenstein, Remarques mêlées, op. cit., p. 67 ; Vermischte Bemerkungen, op. cit., p. 33 [1931].

24. Wittgenstein se démarque de ce point de vue de Kierkegaard. Cf. M. Drury, Conversations avec Ludwig Wittgenstein, op. cit., p. 62 : même s'il n'a jamais discuté de cela avec lui, Drury pense que Wittgenstein n'aurait pas apprécié l'usage fréquent, chez Kierkegaard, des termes " paradoxe » et " absurde », au motif qu' " [i]l faut certainement y voir un effort pour franchir la barrière du langage ». Cela ne doit pas toutefois faire oublier la proximité de vue entre Wittgenstein et Kierkegaard - concernant, notamment, la dimension passionnée, vitale, de la foi ; cf. sur ce point L. Wittgenstein, Remarques mêlées, op. cit., p. 118 ; Vermischte Bemerkungen, op. cit., p. 102 [1946] : «Une des leçons du christianisme, à ce que je crois, est que toutes les bonnes doctrines ne servent à rien. C'est la vie qu'il faut changer [Man müsse das Leben ändern]. (Ou l'orientation de la vie [die Richtung des Lebens]). [Cette leçon est encore] que toute sagesse [alle Weisheit] est froide; et qu'il est tout aussi impossible de redresser sa vie grâce à une sagesse, qu'il l'est de forger le fer à froid. Une bonne doctrine en effet ne peut saisir quelqu'un. On peut seulement la suivre, comme on suit l'ordonnance d'un médecin. - Mais ici, il faut que nous soyons saisis par quelque chose et que nous soyons convertis. - (Du moins est-ce ainsi que je comprends les choses.). Si l'on est converti, alors il faut rester converti. La sagesse est sans passion [Weisheit ist leidenschaftslos]. La Foi, en revanche, Kierkegaard la nomme une passion [eine Leidenschaft]. La religion est pour ainsi dire comme le plus profond de la mer, qui est et reste calme, si haut qu'à la surface puissent s'élever les vagues ».

25. L. Wittgenstein, Carnets de Cambridge et de Skjolden, op. cit., p. 143 ; MS 183, 238-239 [1937]. 
d'une compréhension plus radicale, qui serait le fait d'un homme qui peut employer les concepts, par exemple, de «Christ» ou de «Jugement dernier », dans tous les cas, cela parce qu'il participe pleinement de la forme de vie qui caractérise la croyance religieuse. Wittgenstein note ainsi dans ses Carnets de Skjolden :

Je n'ai pas la foi dans le salut par la mort du Christ; ou en tout cas pas encore. Je n'ai même pas le sentiment d'être sur la voie d'une telle foi, mais il me paraît possible que je comprenne un jour, à ce sujet, quelque chose que je ne comprends absolument pas maintenant, qui ne me dit rien maintenant \& que j'aurai alors une foi que je n'ai pas maintenant ${ }^{26}$.

Citons également un passage des Remarques mêlées, où Wittgenstein démarque le concept de Dieu, qu'il dit comprendre, du concept proprement chrétien de Seigneur qu'il ne comprend pas, du moins à ce moment-là :

Je pourrais [...] appeler [Jésus] «l'Exemple », ou même « Dieu»- ou plus exactement, je puis comprendre quand on le nomme ainsi ; mais je ne puis articuler le mot «Seigneur» avec du sens. Car je ne crois pas qu'il viendra pour me juger, cela ne veut rien dire pour moi. Et cela ne pourrait me dire quelque chose que si je vivais tout à fait autrement [wenn ich ganz anders lebte $]^{27}$.

Je comprends, en un sens plein et entier, les concepts religieux lorsqu'ils jouent, pour ainsi dire, un rôle central dans ma vie. Pour rendre compte de ce rôle, Wittgenstein emploie le terme de «profondeur». La vie du croyant donne ainsi de la profondeur aux phrases :

Une phrase peut paraître absurde \& son absurdité de surface être engloutie par les profondeurs [von der Tiefe] qui, pour ainsi dire, se tiennent sous elle. On peut appliquer cela à l'idée de la résurrection des morts \& à d'autres qui lui sont associées. - Mais ce qui lui donne de la profondeur, c'est l'application [Was ihm aber Tiefe gibt ist die Anwendung] : la vie que mène celui qui la croit. Car cette phrase par exemple peut être l'expression de la plus haute responsabilitée . $^{28}$.

L'idée de profondeur ne renvoie évidemment pas ici à un contenu psychologique quelconque. Il s'agit d'une simple image : la vie donne de la profondeur aux concepts dans la mesure où elle témoigne de l'appropriation par l'individu d'une forme de vie donnée.

26. Ibid., p. 119 ; MS 183, 193-194 [1937].

27. L. Wittgenstein, Remarques mêlées, op. cit., p. 92 ; Vermischte Bemerkungen, op.cit., p. 68 [1937].

28. L. Wittgenstein, Carnets de Cambridge et de Skjolden, op. cit., p. 97 ; MS 183, 147 [1936]. 
Pour Wittgenstein comme pour Luther, la croyance religieuse est, en tout cas, d'abord et avant tout une «affaire de cœur», plus précisément de foi. Le problème qui se pose immédiatement est néanmoins de déterminer comment l'un et l'autre appréhendent cette intériorité.

Il semble, en première approche, que Wittgenstein considère le père de la Réforme comme un tenant du «mythe de l'intériorité ». Au paragraphe 589 des Recherches philosophiques, alors même qu'il emploie le terme biblique de «cœur», il parait en effet « reprocher» à Luther d'envisager la foi comme un état déterminé de l'âme, c'est-à-dire comme un certain contenu psychologique, plutôt que de prendre en considération la pratique de la foi :

« J'ai pris cette résolution du fond du cœur [Ich habe mich in meinem Herzen dazu entschlossen] ». En disant cela, on a tendance à mettre sa main sur la poitrine. Cette façon de parler est à prendre au sérieux du point de vue psychologique. Pourquoi devrait-on moins la prendre au sérieux que l'énoncé qui dit que la foi est un état de l'âme [ein Zustand der Seele] ? (Luther : "La foi se trouve sous la mamelle gauche [unter der linken Brustzitze]) ${ }^{29}$.

Fergus Kerr, dans La Théologie après Wittgenstein, commente ce passage de la façon suivante : "En disant, s'il le dit jamais, que la foi est sous le sein gauche, Luther aurait fait une caricature du mentalisme qui continue de triompher dans le discours chrétien et la piété dans la tradition métaphysique $»^{30}$. Contre le prétendu mentalisme luthérien, Fergus Kerr insiste sur le fait que la localisation corporelle de la foi varie selon les cultures. Il cite à l'appui de cette idée une remarque de Wittgenstein (prise en note par Jackson) extraite de conférences datant de 1946-1947: «Supposons qu'une tribu dise que l'amour est du côté droit de la poitrine, et une autre tribu que c'est dans le côté gauche de la poitrine : s'inquiètera-t-on vraiment beaucoup de savoir qui dit juste? Mais nous nous ferions $d u$ souci au sujet de "l'amour est dans le nez" ${ }^{31 "}$ ". Toute localisation de la foi ne sera pas compréhensible pour nous (par exemple, donc, la localisation de la foi ou de l'amour dans le nez). Il faut toutefois garder en tête que la pratique de la foi admet une certaine souplesse : on ne doit pas circonscrire le sentiment religieux dans une région déterminée, qu'elle soit physique ou mentale - une région, qui plus est, privée, à laquelle j'aurais seul (avec Dieu) accès. Si la foi constitue bien, pour Wittgenstein, le centre névralgique de la vie religieuse, elle ne consiste pas en un certain état de l'âme, que je pourrais tenter de montrer, de désigner, pour définir ce que j'entends par « foi » : à

29. L. Wittgenstein, Recherches philosophiques, op.cit., p. 220 (traduction modifiée); Philosophische Untersuchungen, op. cit., p. 250.

30. Fergus Kerr, La Théologie après Wittgenstein. Une introduction à la lecture de Wittgenstein, trad. fr. A. Létourneau, Paris, Éditions du Cerf, 1991, p. 205.

31. Notes prises par A. C. Jackson lors de conférences de Wittgenstein (1946-1947), citées par Fergus Kerr dans La théologie après Wittgenstein, op. cit., p. 205, qui renvoie en note à Garth Hallett, A Companion to Wittgenstein's "Philosophical Investigations", Ithaca et Londres, Cornell University Press, 1977, p. 623. 
strictement parler, la foi religieuse n'est pas autre chose que sa pratique. Elle ne consiste donc pas en une certaine expérience. Ce concept est, toutefois, ambigu. Wittgenstein peut soutenir, en effet, que la foi relève de l'expérience. Néanmoins, il faut toujours entendre par là une pratique, une attitude, une manière de vivre, indissociable d'une forme de vie donnée, partagée avec une communauté de croyants, et instituée - avant moi - par d'autres que moi. La foi est le produit d'une expérience vitale ainsi entendue: elle n'est pas la conséquence d'une expérience au sens de la recherche de preuves - c'est-à-dire de l'expérimentation. Wittgenstein discrimine notamment ces deux sens de l'expérience dans un passage des Remarques mêlées:

C'est la vie qui peut nous éduquer à la croyance en Dieu. Et ce sont aussi les expériences [Erfahrungen]. Non les visions ou d'autres expériences des sens [sonstige Sinneserfahrungen] qui nous montreraient «l'existence de cette essence », mais des souffrances, par exemple, d'un genre tout différent. Et elles ne nous montrent pas Dieu comme une impression sensible montre un objet, pas plus qu'elles ne nous le font conjecturer. Les expériences [Erfahrungen], les pensées - bref, la vie - peuvent seules nous inculquer un tel concept ${ }^{32}$.

Notons pour finir que si la foi ne consiste pas en une expérimentation, mais seulement en une pratique qui s'inscrit dans une forme de vie partagée et instituée, l'introspection ne s'en trouve pas pour autant niée, rejetée. Il est néanmoins essentiel de garder à l'esprit que cette introspection ne consiste pas en autre chose qu'une pratique. Mieux, dans le cas particulier qui nous occupe - celui de la croyance chrétienne -, la pratique de l'introspection occupe une place que l'on dira volontiers centrale: croire consiste notamment à se remémorer ses fautes, ses péchés, à se demander si notre foi en Dieu est solide, si notre vie est bel et bien accordée à cette croyance, etc. Autrement dit : le croyant, selon Wittgenstein, procède bien au dévoilement, au déchiffrement du cœur au sens de Foucault - mais à la condition de ne voir dans ce déchiffrement rien d'autre qu'une pratique. C'est en ce sens que l'on peut parler, au sujet de la croyance religieuse, d'une pratique intérieure. En parlant de pratique de l'intériorité, nous voulons insister sur le fait que l'authentique croyant, selon Wittgenstein, n'est pas celui qui se livre à certaines pratiques au sens strict, c'est-à-dire opère des œuvres déterminées (aller à la messe, au confessionnal, jeûner, etc.), mais celui dont la vie montre qu'il adhère intérieurement à la vérité révélée - étant entendu qu'il faut entendre par là non pas avoir, à tel ou tel moment, tel sentiment, tel état d'esprit particulier, mais donner à sa vie (y compris a posteriori, après être devenu croyant) une couleur, une tonalité particulière par la pratique (au sens large de manière de vivre, non au sens précis d'œuvre) de la foi. Du fait de cette adhésion interne, la "tapisserie» entière de la vie se trouve

32. L. Wittgenstein, Remarques mêlées, op. cit., p. 161 ; Vermischte Bemerkungen, op. cit., p. 161-162 [1950]. 
modifiée, réorganisée; la vie adopte une nouvelle configuration, une nouvelle découpe, un nouveau séquençage: elle se donne un nouveau commencement (l'apparition de la foi) et une nouvelle fin (le Jugement dernier), la pratique intérieure de la foi engageant ainsi, tout particulièrement, un rapport renouvelé à la temporalité.

\section{2. "La théologie comme grammaire".}

Revenons à Luther. Passe-t-il véritablement, aux yeux de Wittgenstein, pour un tenant dudit «mythe de l'intériorité »? À bien y regarder, cela ne va pas du tout de soi. Mieux, Wittgenstein semble puiser dans la tradition théologique inaugurée par Luther une conception démythifiée de l'intériorité du croyant.

Wittgenstein fait référence à Luther, sans le dire, au paragraphe 373 des Recherches philosophiques. Après avoir indiqué, au paragraphe 371, que «[1]'essence [Wesen] est exprimée dans la grammaire » ${ }^{33}$, il précise que « [1]a grammaire dit d'une chose quelle sorte d'objet elle est. (La théologie comme grammaire) $»{ }^{34}$.

Dans les Carnets de Skjolden, Wittgenstein attribue ce dernier énoncé au père de la Réforme :

On s'agenouille \& on élève le regard \& on joint les mains \& on parle \& on dit que l'on parle avec Dieu, on dit que Dieu voit tout ce que je fais ; on dit que Dieu me parle en mon cœur [Gott spricht zu mir in meinem Herzen]; des yeux, de la main, de la bouche de Dieu, mais non d'autres parties du corps: Apprends ainsi la grammaire du mot « Dieu» [j'ai lu quelque part que Luther avait écrit que la théologie est la « grammaire du mot Dieu», des saintes Écritures]. [...] Une question religieuse ne peut être qu'une question vitale [Lebensfrage] ou un bavardage (creux). Ce jeu de langage - pourrait-on dire - ne se joue qu'avec des questions vitales [mit Lebensfragen]. Tout comme le mot "Ä̈e" n'a pas de signification - sinon comme cri de douleur ${ }^{35}$.

Wittgenstein fait peut-être ici référence à Fritz Mauthner, qui met en exergue de son ouvrage Beiträge zu einer Kritik der Sprache une citation de J. G. Hamann : « avec Luther j'ai transformé l'ensemble de la philosophie en grammaire [ich mit Luther die ganze Philosophie zu einer Grammatik mache $]{ }^{36}$.

33. L. Wittgenstein, Recherches philosophiques, op. cit., p. 170 ; Philosophische Untersuchungen, op. cit., p. 189.

34. Ibid., p. 171 ; p. 189.

35. L. Wittgenstein, Carnets de Cambridge et de Skjolden, op. cit., p. 123 (traduction modifiée) ; MS 183, 202-203 [1937].

36. Fritz Mauthner, Beiträge zu einer Kritik der Sprache, Stuttgart, J. G. Cottasche Buchhandlung Nachfolger G.M.B.H., 1901, exergue. 
Que faut-il entendre précisément par là ? Dire que la théologie est une grammaire signifie chez Wittgenstein, contrairement à ce qu'il pourrait sembler de prime abord, qu'elle n'est pas qu'une affaire de mots, et cela en deux sens. D'une part au sens où la grammaire wittgensteinienne dit proprement ce que sont les choses - non pas toutefois à la manière d'un idéalisme linguistique, qui distinguerait deux entités, le langage et l'être, et considèrerait l'une (le langage) comme la structure de l'autre (l'être). Wittgenstein emploie simplement cette formule pour insister sur le fait que la grammaire n'est pas la récollection des appellations des choses, mais exprime précisément (via l'identification de critères) ce que la chose dont on parle est. Décrire la grammaire des concepts, c'est faire beaucoup plus qu'étudier la manière dont différents signes peuvent se voir combinés. Wittgenstein n'étudie pas de «simples » mots : il n'est pas, de ce point de vue, un "simple » philosophe du langage. Il y insiste au paragraphe 370 des Recherches philosophiques : «Il ne faut pas se demander ce que sont les représentations, ni ce qui se passe lorsqu'on se représente quelque chose, mais comment le mot "représentation" est employé. Ce qui ne signifie cependant pas que je voudrais ne parler que de mots [ich nur von Worten reden will]. Car dans la mesure où il s'agit, dans ma question, du mot "représentation", il s'agit aussi d'une question sur l'essence [Wesen] de la représentation $\gg{ }^{37}$.

La grammaire, d'un autre point de vue, n'est pas qu'une affaire de mots dans la mesure où le mot seul n'a pas de signification indépendamment de la pratique, ou du jeu de langage, dans lequel il s'inscrit. Ce second point nous permet d'aller plus loin dans la compréhension du rapport de Wittgenstein à la tradition luthérienne.

L'auteur des Recherches philosophiques semble s'opposer au théologien protestant Karl Barth, au motif qu'il considèrerait la théologie comme une affaire de mots, et non de jeux de langage. Wittgenstein le cite dans une de ses Remarques mêlées:

Ce que je veux dire réellement, c'est qu'il ne s'agit pas [...] des mots [die Worte] que l'on prononce, ou de ce que l'on pense en le fais ant, mais de la différence qu'ils marquent entre différents moments de la vie [den Unterschied, den sie an verschiedenen Stellen im Leben machen]. Comment sais-je que deux hommes visent la même chose, lorsqu'ils disent chacun croire en Dieu ? [...] La théologie qui fait porter ses efforts sur l'emploi de certains termes et certaines phrases, et qui bannit les autres, ne rend rien plus clair (Karl Barth). Elle s'agite en vain, pour ainsi dire, parmi les mots, parce qu'elle veut dire quelque chose et qu'elle ne sait pas l'exprimer. C'est la praxis qui donne aux mots leur sens [Die Praxis gibt den Worten ihren Sinn $]^{38}$.

37. L. Wittgenstein, Recherches philosophiques, op.cit., p. 170 ; Philosophische Untersuchungen, op. cit., p. 188-189.

38. L. Wittgenstein, Remarques mêlées, op.cit., p. 160-161; Vermischte Bemerkungen, op. cit., p. 161 [1950]. 
Wittgenstein a peut-être en vue ici le projet barthien d'élaboration d'une Dogmatique ${ }^{39}$. Dans la première partie de l'ouvrage, Barth souligne en effet que «[d]ans le matériau brut de la dogmatique, le premier objet est une série d'expressions qui, avec plus ou moins de constance et d'emphase, constituent la matière parlée habituelle de la proclamation dans l'ensemble de l'Église... Mais ici comme partout, ces expressions acquièrent leur sens des associations et contextes dans lesquels elles ont été utilisées ${ }^{40}$. Wittgenstein a (ou aurait) sans doute été sensible ici à la mention du contexte. La théologie, toutefois, ne doit pas consister en l'étude des mots ou expressions mais des pratiques, des attitudes, des manières de vivre ${ }^{41}$.

Wittgenstein serait peut-être plus proche d'un autre théologien protestant du XX $\mathrm{XX}^{\mathrm{e}}$ siècle : Rudolf Bultmann. À notre connaissance, il ne le cite jamais. Cyril Barrett, dans son ouvrage Wittgenstein on Ethics and Religious Belief, indique toutefois que Wittgenstein l'a très certainement $\mathrm{lu}^{42}$. Bultmann envisage la croyance religieuse comme une praxis déterminée. Cela le conduit à démythifier l'intériorité du croyant : l'intérieur (c'est-à-dire l'esprit, à distinguer de la chair) ne consiste pas, selon Bultmann, en une certaine chose, un certain étant - mais en une attitude, une manière de se com-porter, de se tenir dans le monde. Bultmann donne notamment un sens qu'on pourra dire existentiel aux formules pauliniennes «vivre selon la chair» et "vivre selon l'esprit» dans sa conférence Nouveau Testament et mythologie:

Que signifie «chair» ? Ce n'est pas la dimension corporelle et sensible, mais la sphère du visible, du présent [Vorhanden], du disponible, du mesurable, et à ce titre aussi la sphère de l'éphémère. Cette sphère devient une puissance pour l'homme qui en fait le fondement de sa vie, qui vit «d'après elle ", c'està-dire qui se laisse séduire et qui vit du visible, du disponible [...]. Au contraire, la vie authentique de l'homme serait une vie qui vit de l'invisible, de l'indisponible, qui donc renonce à toute sécurité dont l'homme se dote luimême. C'est justement cela, la vie «d'après l'Esprit», la vie « dans la foi ». [...] La démondanéisation n'est fondamentalement pas une ascèse mais une

39. Nous suivons ici F. Kerr, La théologie après Wittgenstein, op. cit., p. 208.

40. Karl Barth, Dogmatique, vol. 1, Première partie, Genève, Labor et Fides, 1953 ; cité par F. Kerr dans La théologie après Wittgenstein, op. cit., p. 208.

41. Pour le rapport à Barth, cf. également M. Drury, Conversations avec Ludwig Wittgenstein, op. cit., p. 115 : en 1930, tandis que Drury lit à Wittgenstein un texte de Barth, l'auteur des Recherches philosophiques dit : «Je ne veux pas en entendre davantage. La seule impression que j'en retire est celle d'une considérable arrogance ». Cf. également ibidem, p. 160 : dix ans plus tard, Wittgenstein, qui est en train de lire Barth, admet que son écriture « a probablement pour origine une remarquable expérience religieuse »; mais après que Drury lui a rappelé l'épisode précédent, il ne mentionne plus Barth.

42. Cf. Cyril Barrett, Wittgenstein on Ethics and Religious Belief, Oxford, Basil Blackwell, 1991, p. 192 ; cité par Gerhard Schmezer dans «Wittgenstein, lecteur de la Bible», Archives de philosophie 2013/1 (Tome 76), p. 123. Pour une tentative de rapprochement entre Wittgenstein et Bultmann, cf. Tim Labron, Bultmann Unlocked, Londres, T\&T Clark, 2012. 
distance par rapport au monde pour laquelle toute participation à ce qui est du monde [das Weltliche] s'accomplit dans l'attitude du «comme si pas » (hôs mê ; 1 Co 7, 29-31) ${ }^{43}$.

La compréhension bultmannienne de la vie selon l'esprit, il est important de le noter, est très proche de celle de Heidegger ${ }^{44}$. Celui-ci procède, tout comme Bultmann, à une démythification de l'intériorité. À la fin du cours qu'il consacre à Paul en 1920-1921, au moment d'évoquer le motif de l'homme intérieur, Heidegger refuse en effet de l'interpréter comme une certaine composante de l'homme, comme un étant déterminé ${ }^{45}$. L'esprit et la chair désignent selon lui des dispositions, des tendances de la vie, c'està-dire des manières de se comporter, de se tenir en elle :

Chez Paul, pneûma a une connexion avec anakrinein et eraunân (1 Co. 2, 15; cf. encore : 2 Co. 4, 16, l'homme «extérieur» et l'homme « intérieur» $\left[\right.$ der ,,äußere“ und ,innere" Mensch $\left.^{46}\right] ; \mathrm{Rm} \mathrm{8,4s,} \mathrm{pneûma} \mathrm{-}$ sarx). Sarx est un phronèma $(8,6)$, une disposition [Gesinnung $\left.{ }^{47}\right]$; c'est-à-dire

43. Rudolf Bultmann, Nouveau Testament et mythologie, trad. J.-M. Tétaz, Genève, Labor et Fides, 2013, p. 70-73. Pour un exposé détaillé de la conception bultmannienne de la chair et de l'esprit, cf. André Malet, Mythos et Logos. La pensée de Rudolf Bultmann, Genève, Labor et Fides, 1962, p. 31-41.

44. On sait que Heidegger et Bultmann furent collègues à l'université de Marbourg au début des années 1920. Sur le couple Bultmann-Heidegger, cf. notamment Sylvain Camilleri, Phénoménologie de la religion et herméneutique théologique dans la pensée du jeune Heidegger. Commentaire analytique des Fondements philosophiques de la mystique médiévale (1916-1919), Dordrecht, Springer, 2008, p. 52-57 ; ainsi que André Malet, Mythos et Logos, op. cit., p. 277-311. Dans le passage de la conférence Nouveau Testament et mythologie cité supra, la proximité avec les interprétations de Heidegger est particulièrement patente. On retrouve notamment chez les deux auteurs une compréhension similaire du hôs $m e ̂$, «comme si pas » paulinien. Cf. pour Heidegger Phénoménologie de la vie religieuse, trad. J. Greisch, Paris, Gallimard, 2012, p. 130-137 ; Ga.60, p. 116-122 (le signe Ga. suivi d'un numéro renvoie aux différents tomes de l'édition complète - Gesamtausgabe - des œuvres de Heidegger en cours de parution chez Vittorio Klostermann à Francfort-surle Main).

45. Nous suivons à partir de là, et jusqu'au terme de l'article, les analyses extrêmement fouillées de Sylvain Camilleri dans Heidegger et les grandes lignes d'une phénoménologie du christianisme primitif, Cham, Springer, 2017, p. 776 et suivantes.

46. Le verset 2 Co. 4, 16 ne fait pas mention du pneûma. Le rapprochement opéré ici par Heidegger entre l'esprit et l'homme intérieur signe, pour Sylvain Camilleri, l'influence de Luther : «Il ne fait pratiquement aucun doute que Heidegger [...] pens[e] à Luther : le vrai homo internus est forcément homo pneumaticus : car là où il n'y a pas de foi, il n'y a pas d'homme intérieur » (Heidegger et les grandes lignes d'une phénoménologie du christianisme primitif, op. cit., p. 780).

47. D'après Sylvain Camilleri, Heidegger suit sans doute ici le Kyrios Christos (1913) de Wilhelm Bousset: "Bousset est à notre connaissance le seul à traduire phronèma par Gesinnung, terme lui permettant justement de distinguer la conception paulinienne de la sarx et du pneûma des conceptions populaires. [...] Bousset montre avec beaucoup d'intelligence comment le pneûma n'est plus, suivant la conception "enthousiaste" d'alors, cette force de Dieu qui va et vient dans l'individu, mais un Esprit habitant littéralement en lui et le soutenant constamment dans l'accomplissement de sa vie religieuse. » (Heidegger et les grandes lignes 
une tendance [Tendenz] de la vie. [...] Chez Paul, il n'y a pas de pneûma einai [être esprit] (comme c'est le cas dans le Corpus Hermeticum), mais un pneûma echein, en pneumati peripatein [avoir l'Esprit, marcher dans l'Esprit], ou epiteleîsthai. Il est donc faux de prendre pneûma pour une composante de l'homme [einen Teil des Menschen]; au contraire, l'anthropos pneumatikos [l'homme spirituel], est un homme qui s'est approprié une certaine spécificité [Eigenheit] de la vie ${ }^{48}$.

Heidegger vise dans ce passage les interprétations qui tentent d'établir un lien entre les écrits pauliniens et les religions à mystères hellénistiques. Il fait référence en note aux analyses de Reitzenstein, dans Die hellenistischen Mysterienreligionen nach ihren Grundgedanken und Wirkungen. Reitzenstein considère que la notion paulinienne d'esprit est héritée du Corpus Hermeticum, qui procède à une identification du pneuma echein au pneuma einai. Dans cette perspective, l'apôtre considérerait donc l'esprit comme une certaine chose à laquelle je suis appelé à m'assimiler - cette chose étant, par ailleurs, radicalement distincte du monde, et m'obligeant donc à rompre absolument avec celui-ci ${ }^{49}$. Heidegger s'oppose fermement à ce type d'analyses ${ }^{50}$ : avoir l'esprit en soi ne signifie pas devenir une certaine chose que je n'étais pas auparavant, mais «simplement» adopter une certaine attitude dans ce monde, vivre d'une nouvelle manière, qui tranche radicalement avec la manière dont les hommes existaient avant la manifestation du Christ ${ }^{51}$.

d'une phénoménologie du christianisme primitif, op. cit., p. 784). S. Camilleri remarque en outre que Bousset et Heidegger s'inspirent peut-être ici de la traduction de Luther $(\mathrm{Rm} 8,6)$ : «Aber fleischlich gesinnt sein ist der Tod, und geistlich gesinnt ist Leben und Friede ».

48. M. Heidegger, Phénoménologie de la vie religieuse, op. cit., p. 138-139; Ga. 60, p. 124.

49. Cf. notamment ce passage du treizième traité du Corpus Hermeticum: "Voyant en moimême une vision immatérielle, produite par la miséricorde de Dieu, je suis sorti de moi-même pour entrer dans un corps immortel et je ne suis plus maintenant ce que j'étais, mais j'ai été engendré par l'Intellect [...]. C'est pourquoi aussi je n'ai plus souci de cette première forme composée qui fut la mienne. Je n'ai plus de couleur, ni le sens du toucher, ni de mesure de l'espace, tout cela m'est étranger » (Corpus Hermeticum, XIII, 3 ; cité par S. Camilleri dans Heidegger et les grandes lignes d'une phénoménologie du christianisme primitif, op. cit., p. 790).

50. Il développe longuement un peu plus haut : « De par son essence propre, le "savoir" exige le pneûma echein. Dans l'exégèse moderne, on s'est livré à des recherches sur les significations du mot pneûma dans la littérature contemporaine de Paul et dans la littérature antique antérieure, en remontant jusqu'à Platon. On perçoit notamment des analogies dans certains passages d'"Hermès Trismégiste" (dans ce qu'on appelle "Corpus Hermeticum"). Du point de vue linguistique-stylistique et temporel, ces passages recoupent ceux de Paul. On prétend que dans le passage cité (1 Co. 2, 10s), Paul se caractérise lui-même comme homme "pneumatique". C'est l'homme lui-même qui deviendrait Dieu. Anèr pneumatikos serait ce qu'il y a de divin en l'homme, anèr psuchikos, ce qu'il y a d'humain en lui. Ce passage a servi d'argument pour établir un lien entre les écrits pauliniens et les religions à mystères hellénistiques. Mais c'est là une erreur » (M. Heidegger, Phénoménologie de la vie religieuse, p. $138 ;$ Ga. 60, p. 123).

51. Cf. Sylvain Camilleri, Heidegger et les grandes lignes d'une phénoménologie du christianisme primitif, op. cit., p. 780: «L'homme intérieur paulinien n'est pas un homo 
Heidegger suit probablement ici (en partie) les interprétations développées par Jülicher dans «Der Brief an die Römer » (1907). Jülicher y explique qu'en distinguant l'esprit et la chair, Paul ne développe pas une analyse métaphysique, mais appelle les hommes à la décision. Dans la deuxième partie de l'article "Fleisch und Geist», auquel Heidegger se réfère en $G a .63$, p. 111, Jülicher insiste également sur le fait que Paul ne se livre pas à un rejet de la chair, mais invite à repenser notre rapport à elle après l'événement de la manifestation du Christ ${ }^{52}$. C'est la raison pour laquelle, peut-on ajouter ${ }^{53}$, Paul prend bien soin d'accoler au terme « spirituel » ou « intérieur » celui d' ' homme »: en croyant, je ne deviens pas l'esprit, mais un homme spirituel; je ne deviens pas quelque chose, je «m'ai » proprement, authentiquement ${ }^{54}$.

Le recours aux notions d'homme intérieur, d'esprit ou de cœur n'engage pas ipso facto l'hypostase ou la réification de l'intériorité. La vie selon l'esprit, c'est-à-dire selon la foi, n'est pas nécessairement tributaire de l'apparition en nous - dans la «région psychique», notre «monde intérieur» - d'un état déterminé de l'âme : elle peut consister en la « simple » adoption d'une attitude, d'un comportement au sein du monde. La démythification de l'intérieur ne vaut pas condamnation pour les pratiques

spiritualis se dressant contre sa sensibilité, tout comme l'homme extérieur n'est pas l'homo sensualis dont l'Esprit devrait l'écarter. [...] L'homme intérieur n'est pas celui qui, dans la gnose, cherche à fuir le monde vers une réalité séparée par et dans l'extase, mais celui qui s'y retrouve et s'y oriente effectivement par la foi et l'espérance d'être prochainement réuni à Christ. L'homme intérieur, c'est celui qui s'approprie toujours plus sa zôe. Son pneûma est pneûma tês pisteôs $(2 \mathrm{Co} 4,13)$ : il lui donne de mieux respirer la vie, et non d'enrôler celle-ci dans un processus magique l'amenant à sortir d'elle-même, plus exactement à s'élever au-dessus d'elle-même ».

52. Pour les analyses de Jülicher, cf. S. Camilleri, Heidegger et les grandes lignes d'une phénoménologie du christianisme primitif, op. cit., p. 781-784. Jülicher opère également une distinction précieuse entre vivre dans la chair (im Fleisch) et selon la chair (nach dem Fleisch) : je peux vivre dans la chair sans pour autant vivre selon la chair - ce qui tend à montrer que la chair (et l'esprit) ne constituent pas (ou en tout cas pas seulement) une certaine chose, un étant déterminé.

53. Avec J. Weiss, dans Der erste Korintherbrief (1910), auquel se réfère S. Camilleri : «Coïncidence ou pas, J. Weiss est le premier à s'être arrêté sur le point mis en lumière par Heidegger, sans toutefois, pour sa part, s'opposer à Reitzenstein. Lui pense que l'absence d'anthrôpos en 1 Co 2,14-15 et 3, 1 (mais aussi en 1 Co 14, 37 ou Ga 6,1) est parfaitement voulue : elle vise paradoxalement à renforcer l'humanité de l'homme contre une prétendue divinité » (Heidegger et les grandes lignes d'une phénoménologie du christianisme primitif, op. cit., p. 792).

54. Notons que dans l'exposé qu'il consacre à Luther en 1924 (prononcé dans le cadre d'un séminaire portant sur l'éthique de Paul, organisé par Bultmann) Heidegger met également en garde ses auditeurs contre l'identification de l'esprit ou de l'homme intérieur à une certaine chose, un certain étant. Ainsi explique-t-il que l'homme devant Dieu - c'est-à-dire l'homme intérieur - renvoie à un comment, un Wie, de l'existence, non à un quoi, à un Was : «L'objet de la théologie, c'est Dieu; son thème est l'homme dans le Comment [Wie] de son êtredisposé [Gestelltsein] devant Dieu» («Le problème du péché chez Luther», traduction par Ch. Sommer, in Le Jeune Heidegger (1909-1926). Herméneutique, phénoménologie, théologie, S.-J. Arrien et S. Camilleri (éds.), Paris, Vrin, 2011, p. 259). 
de l'intériorité : elle entend, tout à l'inverse, en rendre raison, c'est-à-dire libérer un espace pour ces manières de vivre. Cela, Wittgenstein l'a bien compris - et avant lui Bultmann et Heidegger.

L'auteur des Recherches philosophiques n'est évidemment pas un disciple de ces derniers : sa compréhension de la vie religieuse ne consiste pas en un décalque de la leur. Cela principalement parce que les entreprises bultmannienne et heideggérienne de démythification de l'intérieur mettent en jeu le concept d'intentionnalité qui, selon le second Wittgenstein, découle d'une mécompréhension de la grammaire de l'intention, et reconduit ce faisant à une forme dérivée de mentalisme. La conception wittgensteinienne de la croyance religieuse a pu, toutefois, être inspirée par un certain courant de la théologie protestante qui s'est donné pour tâche une certaine démythification de l'intériorité, ouvrant ainsi la voie à la grammaire du cœur. 